Review Article

\title{
Up-to-Date Tailored Systemic Treatment in Pancreatic Ductal Adenocarcinoma
}

\author{
Caroline Y. K. Fong, Emma Burke, David Cunningham, and Naureen Starling
}

The Royal Marsden NHS Foundation Trust, Downs Road, Sutton SM2 5PT, UK

Correspondence should be addressed to Naureen Starling; naureen.starling@rmh.nhs.uk

Received 30 May 2019; Accepted 9 August 2019; Published 5 September 2019

Academic Editor: Jan Bures

Copyright (c) 2019 Caroline Y. K. Fong et al. This is an open access article distributed under the Creative Commons Attribution License, which permits unrestricted use, distribution, and reproduction in any medium, provided the original work is properly cited.

\begin{abstract}
Despite intensive research efforts, pancreatic ductal adenocarcinoma is still regarded as an aggressive and life-limiting malignancy. Combination chemotherapy regimens that underpin the current treatment approach in the advanced setting have led to incremental survival gains in recent years but have failed to confer patients with a median overall survival that exceeds 12 months from diagnosis. Research has since focussed on understanding the role and interplay between various components of the desmoplastic stroma and tumour microenvironment, in addition to developing targeted therapies based on molecular features to improve the prognosis associated with this malignancy. This review will summarise the available systemic treatment options and discuss potential methods to refine the resolution of patient selection to enhance responses to currently available therapies. Furthermore, it will explore newer approaches anticipated to come to the fore of future clinical practice, such as agents targeting the DNA damage response and tumour microenvironment as well as immunotherapy-based combinations.
\end{abstract}

\section{Introduction}

Pancreatic ductal adenocarcinoma (PDAC) is the $14^{\text {th }}$ most common malignancy worldwide, with a global incidence of 458,918 cases in 2018 [1]. In comparison, 432,232 deaths were attributed to PDAC, making it the seventh most common cause of cancer-related death worldwide [1].

At present, surgical resection is the sole curative treatment modality but is relevant to only $15 \%$ of patients presenting with a new diagnosis of PDAC who have resectable disease. Even so, current data indicates that patients who undergo curative resection followed by adjuvant chemotherapy have a limited prognosis consisting of a median overall survival (OS) ranging from 28 to 54 months [2-4] and that patients in the United Kingdom have a five-year survival rate of $3.3 \%$, regardless of stage at diagnosis [5]. Treatment within the palliative paradigm consists of chemotherapy, with the most intensive combination achieving a modest median OS of 11.1 months [6].

The poor prognosis associated with PDAC is a culmination of vague symptomatology leading to late presentation, a complex mutational landscape, and a dense desmoplastic stroma with an immunosuppressive tumour microenvironment (TME) that collectively pose challenges in developing and delivering effective systemic treatments. By 2030, PDAC is projected to become the second leading cause of cancer deaths in America, second only to lung cancer [7]. This forecasted statistic reflects the stagnation of progress in PDAC management relative to other cancers despite intense research efforts over recent years and highlights the urgent need for novel approaches that can provide patients with clinically meaningful benefit. Here, we will outline the current standard-of-care in the advanced disease setting and discuss emerging treatment strategies approaching clinical practice.

\section{Current Approach to Systemic Therapy}

For thirty years, the cornerstone of systemic treatment for metastatic PDAC consisted of 5-fluorouracil (5-FU). This was surpassed in 1997, when patients randomised to receive gemcitabine monotherapy demonstrated an improvement in clinical benefit response, a composite measure consisting of pain, performance status, and weight, in comparison to 
TABLE 1: Summary of first- and second-line trials in metastatic pancreatic ductal adenocarcinoma.

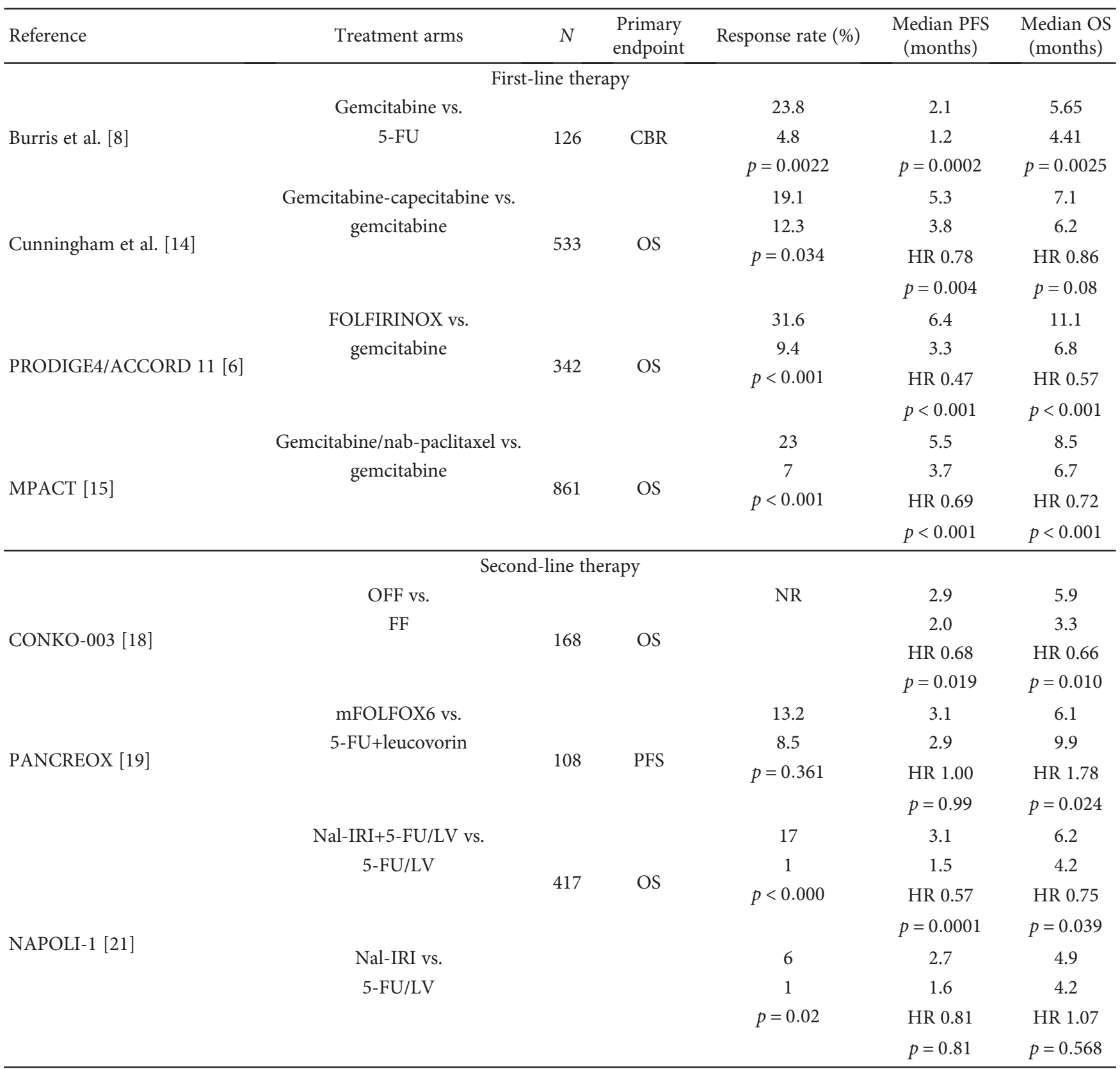

Abbreviations: CBR: clinical benefit response; FF: leucovorin+fluorouracil; HR: hazard ratio; FOLFIRINOX: 5-fluorouracil, irinotecan+oxaliplatin; mFOLFOX6 (modified FOLFOX6): 5-fluorouracil, leucovorin, and oxaliplatin; OFF: oxaliplatin+leucovorin+fluorouracil; LV: leucovorin; Nal-IRI: nanoliposomal irinotecan; OS: overall survival; PFS: progression-free survival; 5-FU: 5-fluorouracil.

patients treated with 5 -FU (23.8\% vs. $4.8 \%, p=0.002)$ [8] (Table 1). Additionally, the gemcitabine group demonstrated a small survival advantage over patients who received 5-FU (median OS 5.65 vs. 4.41 months, $p=0.002$ ).

Clinical trials examining the efficacy of gemcitabinecontaining doublet regimens with a second chemotherapeutic agent or targeted therapies were largely negative [9-12]. There are notable exceptions. Although a phase III randomised trial comparing gemcitabine and erlotinib against gemcitabine alone showed a statistically significant improvement in both progression-free survival (PFS) and OS in the combination arm, this failed to translate clinically as it amounted to an absolute difference of 6 and 10 days, respectively [13]. Interestingly, a subset of patients with grade $\geq 2$ skin rash obtained a more significant survival benefit compared to patients with milder or no skin toxicity (10.5 months vs. 5.8 months vs. 5.3 months, $\operatorname{HR} 0.74, p=0.037$ ). Nevertheless, this combination is rarely utilised in clinical practice as its potential benefit is limited to a small proportion of patients. A randomised controlled trial examining the efficacy of gemcitabine and capecitabine against gemcitabine monotherapy demonstrated statistically significant improved response rates $(19.1 \%$ vs. $12.4 \%, p=0.34)$ and median PFS (5.3 vs. 3.8 months, HR $0.78, p=0.004$ ), with a 
trend towards improved median OS (7.1 vs. 6.2 months, HR $0.86, p=0.08)$ [14]. A meta-analysis of these results amalgamated with data from two further studies detected a survival benefit associated with gemcitabine and capecitabine over monotherapy (HR 0.86 ; 95\% CI, 0.75 to $0.98 ; p=0.02$ ), providing support for the use of this regimen in treatment-naïve advanced PDAC patients [14].

Two further chemotherapy combinations provide treatment options in the first-line setting for advanced PDAC in patients fit for combination therapy. The triplet combination of 5-FU, irinotecan, and oxaliplatin (FOLFIRINOX) was shown to improve median OS to 11.1 months, in comparison to 6.8 months in patients receiving gemcitabine (HR 0.57, $p<0.001$ ), with a parallel improvement in quality of life at 6 months as assessed by the EORTC QLQ-C30 questionnaire (HR 0.47, $p<0.001$ ) [6]. Separately, gemcitabine and nanoparticle albumin-bound paclitaxel (nab-paclitaxel) also extended median OS to 8.5 months, in comparison to 6.7 months with gemcitabine monotherapy (HR $0.72, p<0.0001)$ in patients with metastatic disease (Table 1) [15].

At face value, FOLFIRINOX may be perceived as the superior regimen due to its longer median OS, albeit with a less tolerable safety profile. However, a direct comparison of outcomes between both trials cannot be made due to variations in study methodologies and patient characteristics. A systematic review and meta-analysis of 16 retrospective studies involving 3,813 patients comparing the effectiveness of FOLFIRINOX against gemcitabine/nab-paclitaxel showed that both regimens resulted in similar OS, PFS, overall response rates, and risks of death and disease progression with distinct toxicity profiles [16]. These findings reinforce the notion that both combinations are reasonable upfront treatment options for patients with a good performance status, and the choice of treatment should ultimately be individualised accordingly to patient's comorbidities and preferences. In patients with poor performance status, gemcitabine monotherapy can still be considered in patients treated with palliative intent.

Approximately $40 \%$ of patients eventually receive second-line systemic therapy [17] following gemcitabinebased first-line therapy. There is currently no prospective trial data to determine the optimal sequencing of FOLFIRINOX and gemcitabine/nab-paclitaxel, and these remain as treatment options in patients requiring second-line therapy provided they remain fit for these regimens. Oxaliplatinbased chemotherapy has been considered as standard-ofcare in this setting after oxaliplatin, leucovorin, and 5-FU (OFF) demonstrated superior median OS in the CONKO003 trial when compared to leucovorin and 5-FU (FF) (5.9 vs. 3.3 months, HR $0.66, p=0.001$ ) [18]. These findings were challenged by results from the PANCREOX trial, which showed that survival outcomes were inferior in patients randomised to receive modified FOLFOX6 (mFOLFOX6; 5-FU, leucovorin, and oxaliplatin) in comparison to patients in the 5-FU-leucovorin arm [19]. A higher incidence of grade $\geq 3$ adverse events and adverse events leading to treatment discontinuation was observed in the mFOLFOX6 arm compared to the 5-FU-leucovorin arm (63\% and $20.4 \%$ vs. $11 \%$ and $1.9 \%$, respectively). Furthermore, a higher proportion of patients in the 5-FU-leucovorin arm received postprogres- sion therapy in comparison to patients assigned to receive mFOLFOX6 ( $25 \%$ vs. $6.8 \%$ ), possibly due to the poorer tolerability associated with the former combination. As the median PFS of both arms were similar (HR 1.00, $p=0.36$ ), the discrepancy in OS could be attributed to the differences in postprogression therapy uptake [19]. More recently, long-term data from the NAPOLI-1 trial [20] has since confirmed that nanoliposomal irinotecan and 5-FU-leucovorin is associated with superior median OS when assessed against 5-FU-leucovorin [21], providing a further option for patients with gemcitabine-refractory disease who remain well enough for additional treatment (Table 1). Although therapeutic options for second-line therapy are available, the risk benefit of pursuing further systemic treatment should be carefully considered in view of the significant symptomatic burden and rapid clinical deterioration commonly seen in these patients at this juncture.

\section{Improving Patient Selection to Maximise Treatment Efficacy}

In contrast with other malignancies, targeted therapies do not feature in the treatment armamentarium due to a lack of a targetable driver mutation in this disease. Although over $90 \%$ of PDAC cases harbour activating KRAS mutations [22], thus providing an attractive therapeutic target, KRAS is deemed "undruggable" due to its relatively smooth 3D structure [23]. Efforts to target downstream components of the RAS pathway, such as mitogen/extracellular signalregulated kinase (MEK), have also been unsuccessful in the clinical setting [24-26]. The epidermal growth factor receptor (EGFR) is overexpressed in 80\% [27] of PDACs, which collectively with KRAS mutations may explain the lack of efficacy from anti-EGFR-targeted therapies [13, 28]. Given that there has been a paucity of novel therapeutic agents coming to the fore of PDAC management, research activity has also focussed on devising methods to refine patient selection for treatment based on individual patient molecular profiles. Several molecular characterisations for PDAC exist [29-33] using tissue from both patients with early and advanced disease, and current classifications have identified molecular features with prognostic and responsive associations. For example, the basal subtype is associated with chemoresistance and poor prognosis, whereas the classical subtype is associated with a more favourable outlook [29]. The COMPASS trial was designed as a prospective study to assess the feasibility of comprehensive real-time whole genome sequencing and RNA sequencing of advanced PDAC to identify predictive mutational and translational features to inform treatment selection [34]. In addition to demonstrating that prospective genomic profiling was feasible with a median turnaround time of 35 days, the investigators detected better objective responses to first-line chemotherapy in patients with GATA6-amplified classical PDAC RNA subtype in comparison to basal-like patients $(p=0.004)$, with the best PFS observed in patients who received modified FOLFIRINOX [34]. While this finding provides some prospective evidence in a nonrandomised cohort that the characterisation of RNA subtypes via molecular profiling may allow upfront 
identification of chemosensitivity to potentially guide treatment selection, it requires further assessment in a controlled trial to fully ascertain its role in the clinic.

Another promising method to enhance treatment selection is through the use of patient-derived organoids (PDOs) to facilitate in vitro drug sensitivity testing [35]. PDOs are cultured from $\mathrm{LRG}^{+}$stem cells that can be isolated from a number of organs and propagated as epithelial cyst-like structures in vitro with genomic, transcriptomic, and phenotypic properties resembling those of the original tissues [36]. Vlachogiannis et al. successfully developed PDOs from metastatic lesions of gastrointestinal primaries obtained at baseline and posttreatment time points [36]. Concordance of immunohistochemical markers and genetic aberrations such as ERBB2 and FGFR2 amplifications between PDOs and parental tissue was observed, in addition to a $96 \%$ overlap in mutational spectrums. Furthermore, it was demonstrated that PDOs could predict response to both targeted therapies and chemotherapy and mirrored the development of drug resistance in tandem with clinical outcomes. Similar findings have been reproduced in 66 PDAC PDOs cultured from both tissue obtained from surgical resections and fine needle biopsies [37]. Both these studies highlight that PDOs are robust preclinical models that can provide accurate representation of tumour histopathology and molecular heterogeneity by predicting treatment efficacy in vitro and are posed to be an invaluable tool in future drug development with possible clinical utility in prospective therapeutic selection.

Large-scale efforts to accrue molecular profiles representative of the patient population are essential to better our understanding of this complex disease and identify newer, rational therapeutic options. An example is the PRECISIONPanc platform in the United Kingdom, which serves as a portal protocol for patients with PDAC that offers patients full molecular characterisation of tumour tissue using upfront genomic sequencing with subsequent access to appropriate clinical trials alongside an integrated preclinical development programme [38]. An example of a clinical trial affiliated with the PRECISION-Panc platform is PRIMUS-001, which aims at comparing the efficacy of a novel platinum-containing regimen FOLFOX-A (5-FU, oxaliplatin, and nab-paclitaxel) against gemcitabine and nab-paclitaxel, with an incorporated translational research objective to further identify and characterise biomarkers of response to DNA-damaging agents. By allowing bidirectional translation between the laboratory and the clinic, platforms such as PRECISION-Panc represent an important opportunity to obtain and analyse multidimensional datasets which can enable identification of novel pathophysiology and treatment approaches to inform the direction of future research within the field.

\section{Selected New Treatment Approaches on the Horizon}

4.1. Targeting DNA Damage Repair Signalling. Endogenous and exogenous stressors such as reactive oxygen species and cytotoxic agents continuously produce DNA damage. This triggers a coordinated response orchestrated by an intricate network of DNA damage response (DDR) proteins to main- tain genomic integrity and sustain cell viability. Consequently, defective DDR is associated with susceptibility to cancers including PDAC, ovarian, breast, and prostate cancers $[39,40]$. The prevalence of a deleterious germline mutation in BRCA1 and BRCA2, which encode for mediators of homologous recombination (HR), is estimated at $5 \%$ in PDAC patients [41], while aberrations in PALB2 [42], ATM [43], ATR [44], RAD51 [45], and CHK1/2 [44] genes can also confer a HR-deficient or BRCA-like phenotype. Additionally, approximately $14 \%$ of PDACs have large numbers of structural variation events, resulting in genomic instability and DDR deficiency [31].

Impaired DDR signalling has classically been targeted by DNA-damaging agents such as platinums and alkylating agents. Agents interfering with DNA repair, most notably polyADP-ribose polymerase (PARP) inhibitors, are currently being assessed in PDAC. Clinical evaluation of PARP inhibitors in combination with chemotherapy (NCT01585805) is underway. Recently, results from the POLO study demonstrated that patients with germline BRCA-mutated metastatic PDAC who achieve disease control following a minimum of 16 weeks of platinum-based first-line chemotherapy reported a statistically significant improvement in PFS (7.4 months vs. 3.8 months, HR 0.53 , 95\% CI 0.35 to $0.82 ; p=0.004)$ when randomised to receive olaparib over placebo. Although an OS benefit was not detected, these results provide a basis for the potential clinical application of a new class of therapeutics in a novel clinical setting within pancreatic cancer management [46]. Beyond BRCA mutant patients, HR-deficient patients represent another population who could derive benefit from agents that target DNA damage repair signalling.

\subsection{Understanding the Desmoplastic Stroma and Immune} Microenvironment in Pancreatic Ductal Adenocarcinoma. PDACs are encased by a desmoplastic reaction, which forms a fibrous layer of tissue surrounding malignant cells in both primary and metastatic lesions [47]. This consists of a diverse network of cellular components, including cancer-associated fibroblasts (CAFs), endothelial cells, immune cells, and extracellular matrix. The majority of these cells are CAFs, which secrete extracellular matrix proteins such as collagen, proteoglycans, fibronectin, matrix metalloproteases, and glycosaminoglycans [48]. While the resultant stroma is thought to be integral in promoting cancer growth and metastasis in addition to forming a physical barrier that facilitates a hypoxic environment and limits drug penetration [49], there is an increasing body of evidence to suggest that CAFs may restrain rather than promote PDAC tumour growth $[50,51]$. Recent observations demonstrating significant intra- and intertumoural heterogeneity in patient-derived CAF primary cultures have provided insight into the complexity of CAFs and its role in the desmoplastic stroma [52] and support the hypothesis that CAFs play a context-dependent role in PDAC tumorigenesis and progression.

Although PDAC has a relatively low mutational load with a median somatic mutational prevalence of 1 mutation/megabase [53], the majority of PDACs express candidate neoantigens required to generate an antitumour response 
[54]. Additionally, it has been shown using in silico neoantigen prediction that tumours with the highest number of neoantigens alongside the most abundant CD8+ T-cell infiltrates are associated with the longest survival [55]. The presence of dendritic cells, a form of antigen-presenting cell required to stimulate a $\mathrm{T}$-cell response, is infrequent in PDAC and tends to be immature even when present, compromising tumour antigen recognition and T-cell activation [56]. While PDAC tumour samples also demonstrate robust presence of tumour-infiltrating lymphocytes, active immune suppression mechanisms mediated by the presence of $\mathrm{T}$ regulatory cells, myeloid-derived suppressor cells (MDSCs), tumour-associated macrophages (TAMs), and inhibitory cytokines such as TGF- $\beta$, IL-10, and nitric oxide synthase mitigate against effective T-cell responses [54]. In light of these immunosuppressive mechanisms, PDAC is regarded as an immune-quiescent tumour.

The TME permits cross talk between malignant cells, its stromal components, and immune cells, creating a dynamic milieu which evolves throughout the course of the disease. Clearly, understanding the role of the desmoplastic stroma and its immune microenvironment is a crucial step in developing new therapeutic targets in PDAC, improve drug delivery, and broaden the clinical application of immunotherapy in this disease.

4.3. Targeting Components of the Desmoplastic Stroma. Interest was initially focussed on targeting the Hedgehog pathway, a key driver of the desmoplastic process. Its promise was first highlighted when the combination of saridegib, a Hedgehog inhibitor, and gemcitabine induced a transient increase in intratumoural vascular density and intratumoural levels of gemcitabine, resulting in improved survival in a patientderived xenograft murine model [57]. These preclinical observations failed to translate into clinical benefit in early phase clinical studies. Further research has since shown that stromal constituents driven by the Hedgehog pathway act to restrain rather that promote tumorigenesis in PDAC $[51,58]$, highlighting the diverse roles of individual stromal components which subsequently pose challenges to successful drug development.

Focal adhesion kinases (FAKs) are nonreceptor tyrosine kinases that regulate cell signalling within the TME. They are frequently overexpressed in several advanced-stage solid tumours, including PDAC, and are important regulators of the fibrotic and immunosuppressive TME in PDAC when hyperactivated [59]. FAK inhibition has demonstrated antiproliferative therapy in vitro [60] and extended response to gemcitabine and nab-paclitaxel in patient-derived xenograft models [59]. These preclinical observations collectively form the basis of clinical trials incorporating FAK inhibition with trametinib (NCT02428270), gemcitabine (NCT02546531), and pembrolizumab (NCT02758587) (Tables 2 and 3). Preclinical models of solid tumours lacking neurofibromatosis type 2 (NF2) tumour suppressor gene product, merlin, exhibit exquisite sensitivity to FAK inhibition monotherapy [61], a finding that has also been recapitulated in early phase clinical trials [62]. Merlin deficiency is noted in over $40 \%$ of PDAC and is associated with adverse prognostic factors such as higher T stage, increased lymph node disease, and poorly differentiated histology [63]. The integration of predictive biomarkers of response similar to merlin loss could form the rationale for future biomarker-selected trials.

Another TME component that holds potential as a therapeutic target in PDAC is hyaluronan (HA), a glycosaminoglycan found in the stromal matrix. Structurally, HA has multiple anionic repeats that attract cations, resulting in osmotic swelling [64]. Tumours with high levels of HA have higher intratumoural interstitial gel fluid pressure which acts as a barrier to perfusion and reduces drug penetration to cancer cells [65]. When tested in preclinical models, pegylated recombinant human hyaluronidase (PEGPH20) was shown to degrade HA with subsequent normalisation of interstitial fluid pressures and reexpansion of the microvasculature within PDAC tumours [66]. Phase II evaluation has shown that PEGPH20 in combination with gemcitabine and nabpaclitaxel is particularly efficacious in patients with HAhigh tumours, defined as extracellular matrix HA staining $\geq 50 \%$ of tumour surface at any intensity [67], and a phase III trial is ongoing in this subgroup of patients (NCT02715804). Interestingly, PEGPH20 given concurrently with FOLFIRINOX in an unselected group of patients resulted in detrimental OS outcomes and was associated with significantly higher rates of grade $\geq 3$ toxicity [68]. These results highlight the importance of considered selection of individual combination therapy components as the interest in combinatorial strategies intensifies and becomes an increasingly common treatment approach in this tumour type. Efforts to exploit various targets within the desmoplastic stroma such as angiogenesis, hypoxic environment with the TME, and the Wnt- $\beta$-catenin pathway as therapeutic targets have also resulted in negative results to date (Table 2).

\subsection{Extending the Impact of Immunotherapy in Pancreatic} Ductal Adenocarcinoma. Although immune checkpoint inhibition has transformed the disease trajectory of tumour types historically associated with poor outcomes such as melanoma and non-small-cell lung cancer, these results have not been reproduced by both anti-CTLA- 4 and anti-PD-L1 agents as monotherapy $[69,70]$ or in combination in biomarkerunselected populations with PDAC [71]. Immune checkpoint inhibition (ICPi) is only currently indicated in patients with mismatch repair- (MMR-) deficient disease, for which pembrolizumab received tumour-agnostic approval after it was shown to induce responses across multiple tumour types with this phenotype, including PDAC $[72,73]$. The prevalence of MMR deficiency in PDAC is estimated between 1 and 2\% [74], limiting the benefit of immune checkpoint inhibition to this small proportion of patients.

To that end, various combinatorial strategies aimed at priming the immune response in preparation for ICPi therapy have been explored in PDAC. For instance, chemotherapies facilitate dendritic cell recruitment and activation [75] in addition to tumour-specific antigen release [76], and studies evaluating the combination of anti-CTLA4 and anti-PD1/PD-L1 antibodies alongside chemotherapies are ongoing (Table 3).

Other combinations include agents targeting the desmoplastic stroma, such as PEGPH20 and FAK discussed 


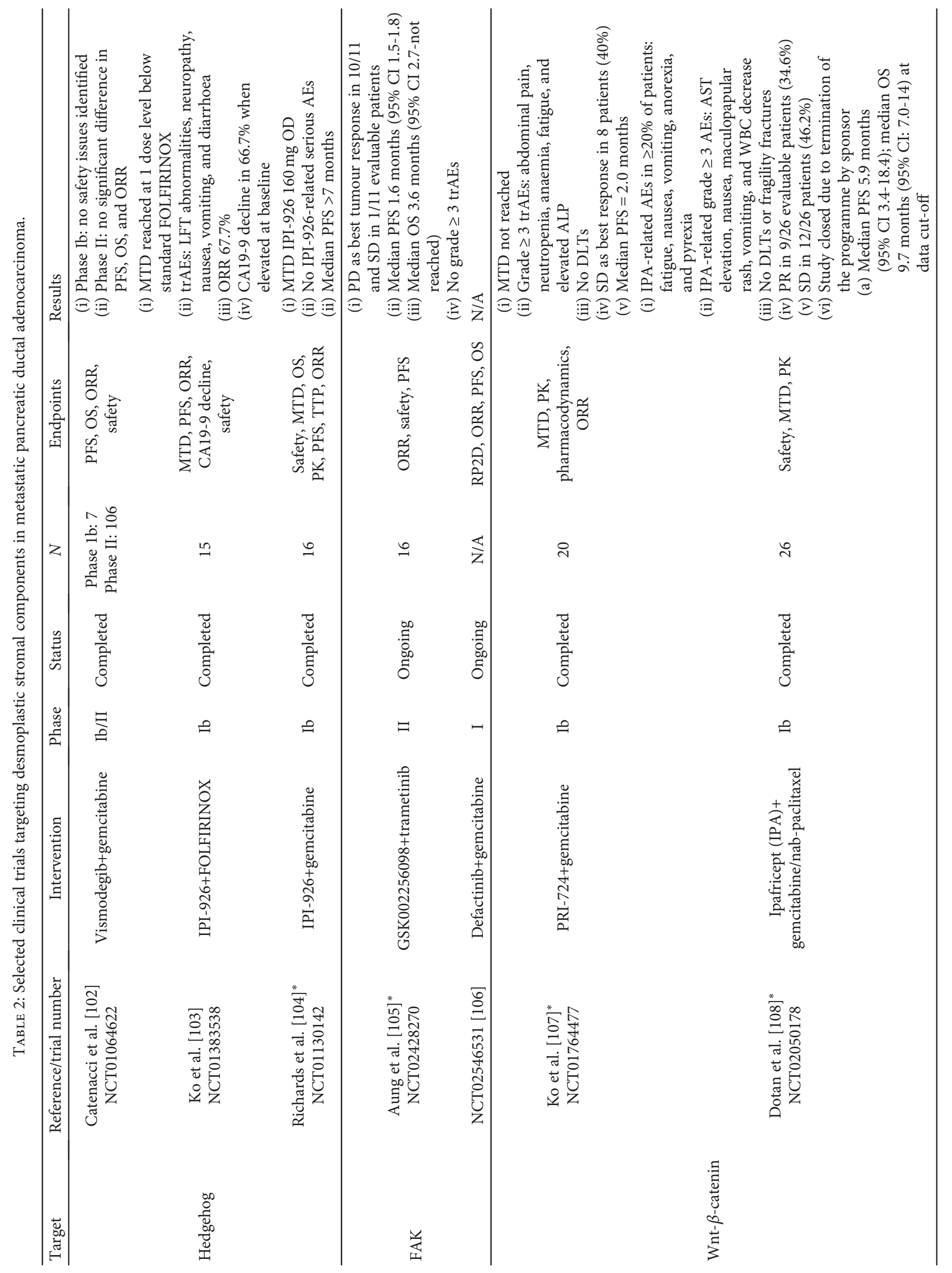




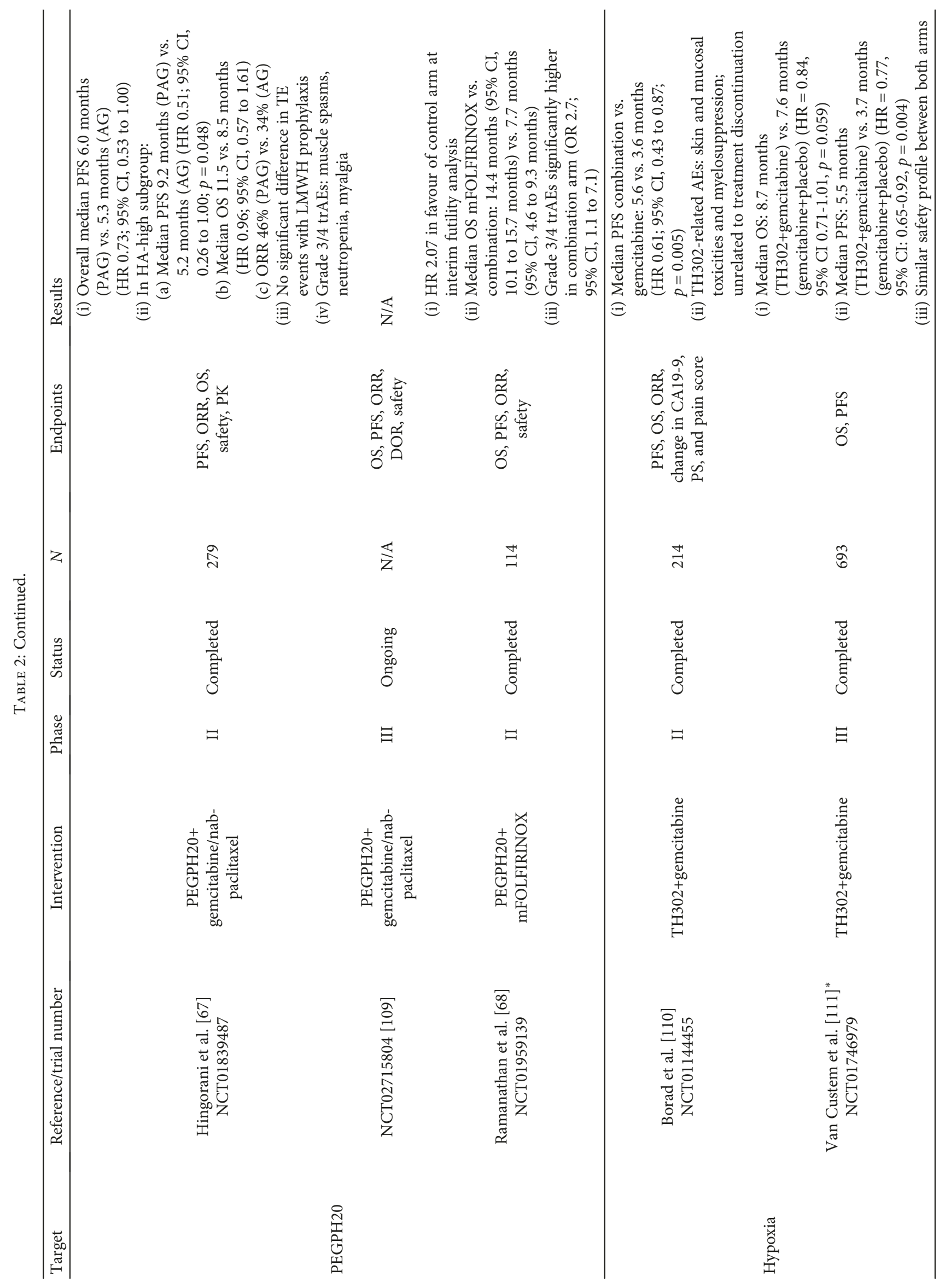




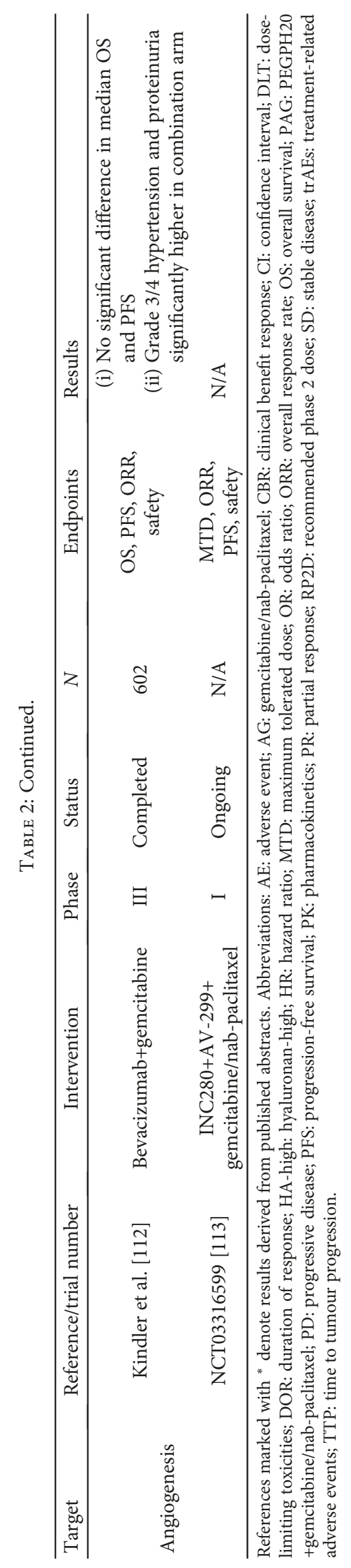




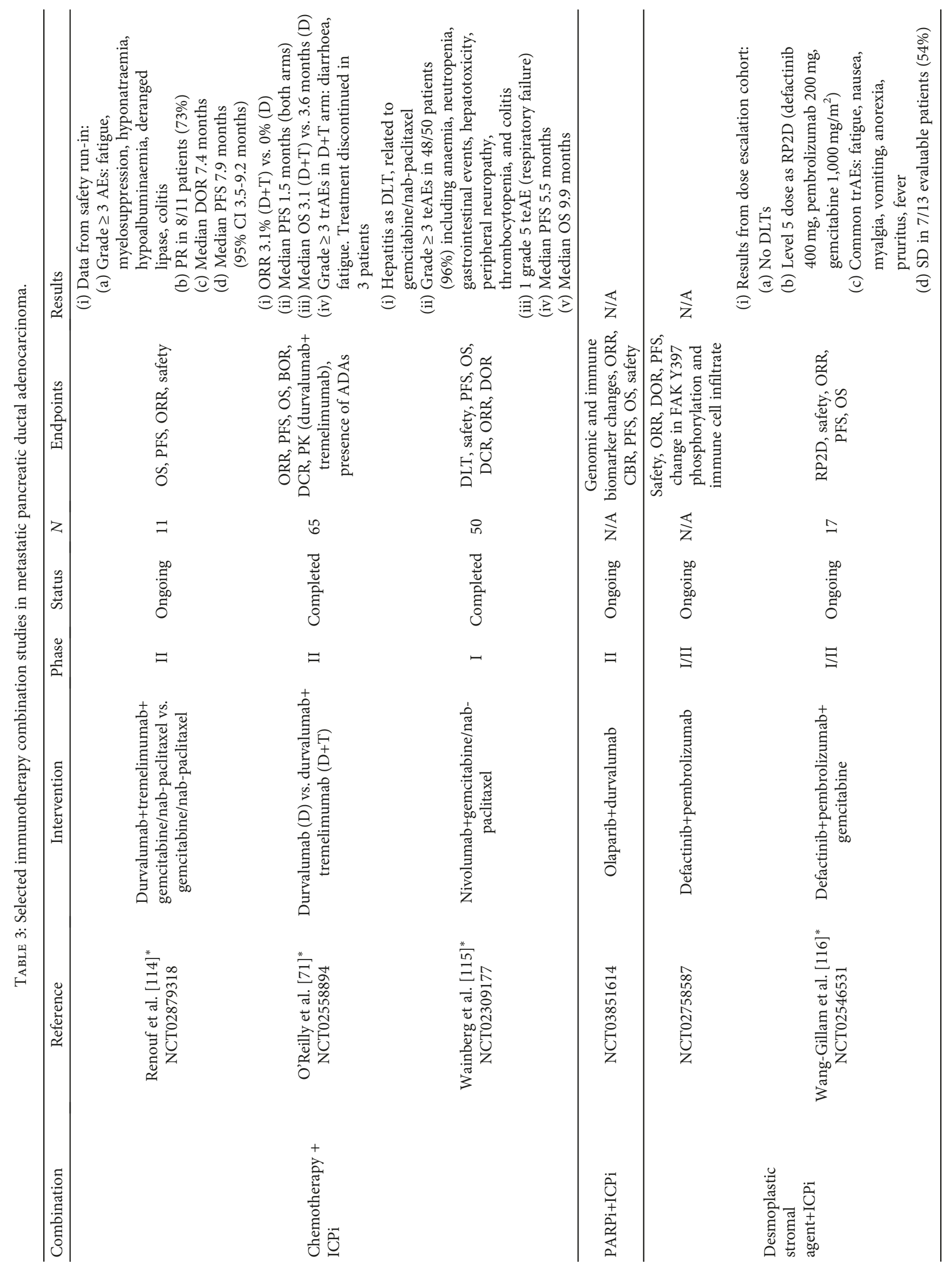




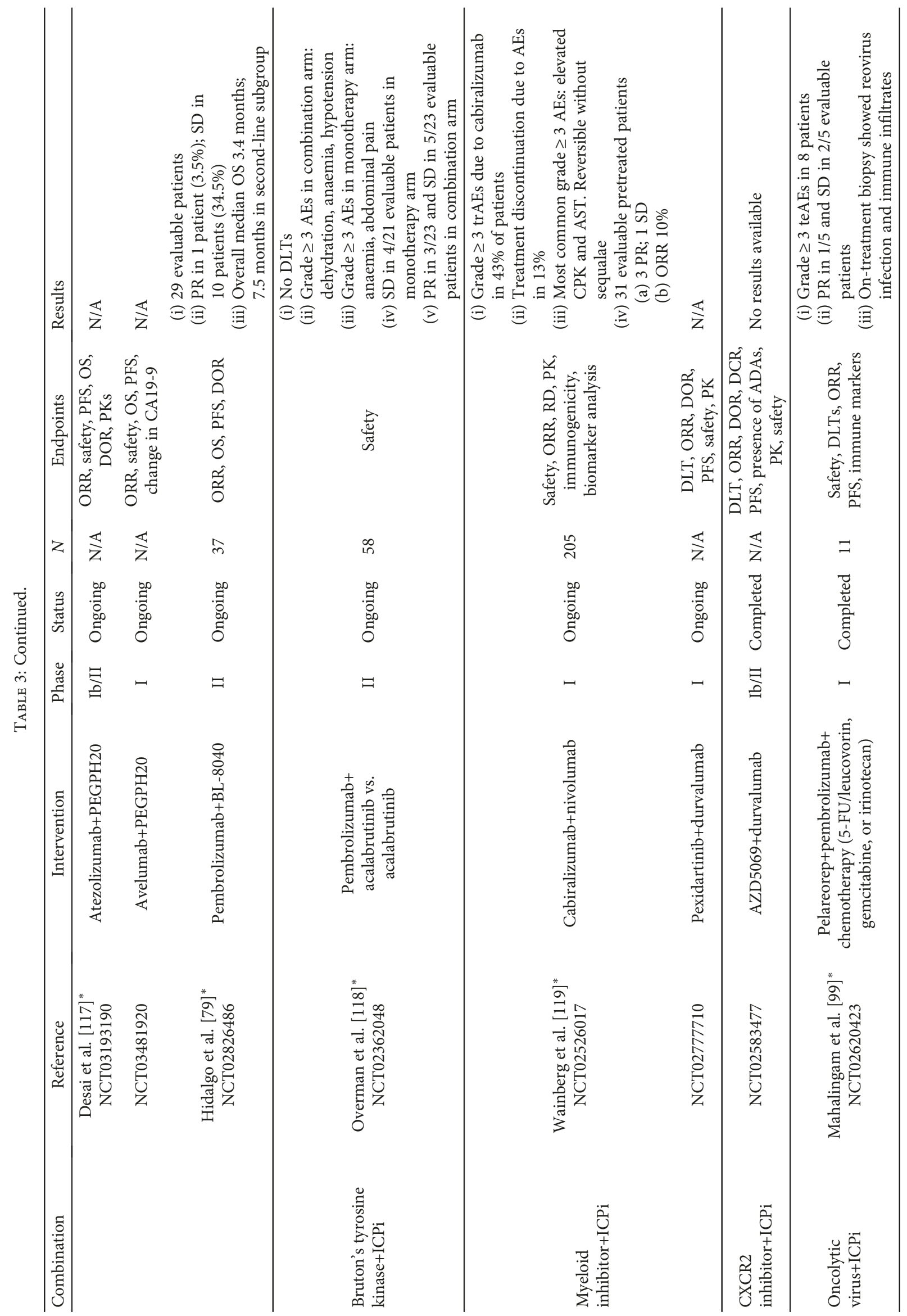




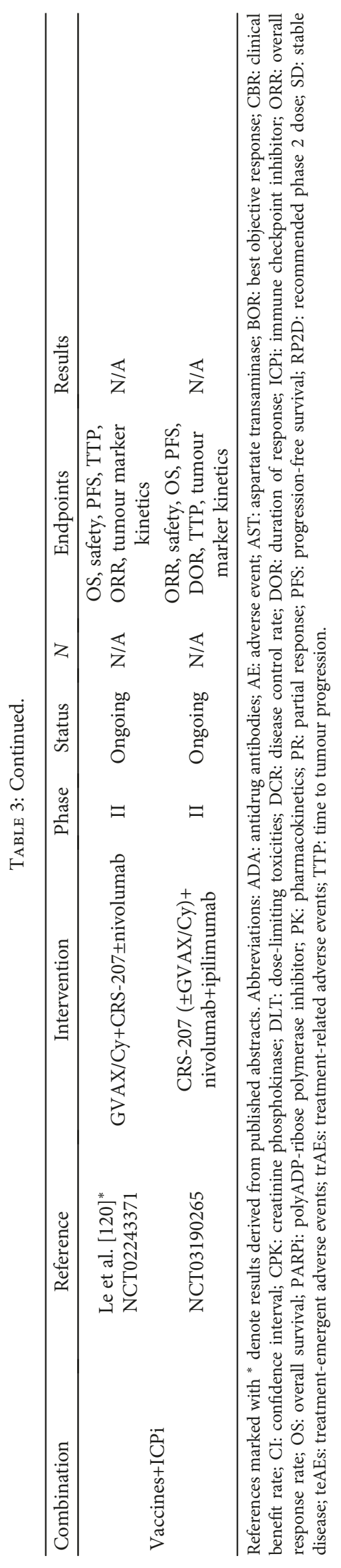


previously. The C-X-C motif chemokine receptor type 4 (CXCR4)/stromal-derived factor-1 (CXCL12) is another candidate for combination with ICPi. Cancer stem cells with strong CXCR4 expression on the invasive front have been found to be a driver of metastatic behaviour and are prerequisite for the development of liver metastases [77]. CXCL12 secreted by CAFs has been shown to mediate immunosuppression, and its inhibition with AMD3100 induced rapid T-cell accumulation and synergistic activity with an antiPD-L1 therapy in a PDAC mouse model [78]. Clinically, a phase II trial is underway with early results reporting that BL-8040 with pembrolizumab achieved a median OS of 7.5 months in patients receiving this as second-line therapy [79] (NCT02826486, Table 3).

As myeloid cells such as TAMs and MDSCs are important mediators of immune evasion, these have been identified as potential therapeutic targets in PDAC in the hope of overcoming its innate immunologically resistant phenotype. For example, C-C chemokine receptor type 2 (CCR2) and colony-stimulating factor-1 receptor (CSF1R) are involved in the recruitment and differentiation of TAMs within the PDAC TME. CSF1R or CCR2 inhibition has been shown to reduce the numbers of pancreatic tumour initiating cells and improve chemotherapy efficacy [80], and CSF1R inhibition upregulates PD-L1 and CTLA-4 checkpoint molecules in response to ICPi [81]. CSF1R inhibitors are currently being evaluated in combination with immunotherapy and chemotherapy (NCT02526017, NCT02777710) (Table 3).

Newer immune targets such as the CXCR2 axis are also being evaluated with ICPi. The primary role of the CXCR2 axis is to regulate neutrophil migration to the site of inflammation [82], and CXCR2 signalling at the tumour border is a poor prognostic indicator in human PDAC [83]. CXCR2 inhibition has been found to augment T-cell entry and increase sensitivity to ICPi therapy when used in combination in a mouse model [84], providing a rationale to pursue clinical testing (NCT02583477) (Table 3).

Another target thought to have immunomodulatory capabilities in PDAC is Bruton's tyrosine kinase (BTK), a Tec family nonreceptor tyrosine kinase that is required for B-cell receptor signalling. The BTK inhibitor, ibrutinib, has also been shown to exert antifibrotic effects on the desmoplastic stroma through inhibition of mast cell activity [85], as well as amplify cytotoxic T-cell activity with subsequent enhancement of responsiveness to chemotherapy in PDAC murine models [86]. Despite this encouraging preclinical data, a phase III trial randomising patients between gemcitabine/nab-paclitaxel in combination with ibrutinib or gemcitabine/nab-paclitaxel with placebo as first-line treatment of metastatic PDAC failed to meet its primary endpoints of PFS and OS (NCT02436668) [87] . Acalabrutinib is currently being assessed in combination with pembrolizumab at the phase 2 level, with preliminary results indicating an acceptable safety profile and encouraging antitumour activity in a pretreated PDAC patient population (NCT0236048) (Table 3).

Various types of vaccine therapies are also in active development. Personalised peptides designed to prevent progressive tolerance to cancer-related antigens given concurrently with gemcitabine have also shown promise [88] but require further investigation to establish its efficacy and development into a deliverable treatment modality. Another vaccine-based approach is whole-cell tumour vaccines, which enable multiple antigens to be targeted simultaneously to elicit a more robust T-cell response. Amongst the most studied is GVAX, which is genetically engineered to secrete GM-CSF, a cytokine that mobilises leucocytes to the TME and generates large immunoglobulin (Ig) G and IGM responses [89]. GVAX is thought to be an ideal primer for checkpoint inhibition, as it increases immunogenicity by inducing $\mathrm{T}$-cell infiltration and formation of tertiary lymphoid aggregates [90], as well as upregulates the PD-1/PD-L1 pathway in PDAC patients [91]. Based on preclinical data suggesting that sequential administration of two vaccines to firstly "prime" the immune system then "boost" the immune response achieves synergistic enhancement of T-cell induction, the combination of GVAX and CRS-207, a live-attenuated Listeria monocytogenes vaccine expressing mesothelin, was evaluated at the phase II level. These results demonstrated extended survival of PDAC patients with minimal toxicity with this combination [92]. However, administration of CRS-207/GVAX or CRS-207 alone did not amount to a survival benefit over single-agent chemotherapy in a pretreated metastatic PDAC cohort (NCT02004262) [93]. Ongoing evaluation of its clinical potential in combination with ICPi therapy is currently being investigated (Table 3 ).

Additionally, the modulation of the gut microbiome in PDAC is an emerging field. The cancerous pancreas has been found to harbour distinct and more abundant gut microbiota in comparison to normal pancreatic tissue in both mice and humans [94]. Bacterial ablation using antibiotic therapy has been shown to be protective against oncogenesis, reversed intratumoural immune tolerance, and increased susceptibility to immune checkpoint blockade [94]. On this basis, the effects of metronidazole and ciprofloxacin given with pembrolizumab on immune activation in pancreatic cancer tissue in patients with surgically resectable PDAC will be investigated in an upcoming pilot study (NCT03891979).

A further immunotherapeutic approach is the application of tumour-oncolytic viruses (TOVs) to selectively infect, replicate in, and lyse tumour cells to unleash virions that can subsequently infect adjacent tumour cells, a process that potentiates an inflammatory response through immunogenic cell death [95]. Cell lysis also releases pathogen- and damageassociated molecular pattern molecules which activate the innate immune response, as well as viral- and tumourassociated antigens which stimulate the adaptive immune response [95]. Adenoviruses have been most extensively evaluated thus far. Given alone, ONYX-015, an adenovirus that selectively replicates and lyses in cells with p53 abnormalities, failed to induce objective responses in a phase I dose escalation study following intratumoural injection or exhibit evidence of viral replication [96] but resulted 2 partial responses and disease control in 8 patients when administered alongside gemcitabine in a cohort of 21 patients [97]. Phase II data investigating pelareorep, a reovirus, in combination with gemcitabine induced a partial response in one patient $(n=29)$ and PD-L1 upregulation following treatment 
[98], suggesting an immunomodulatory effect in PDAC and providing a rationale for pursuing combination therapy with immune checkpoint blockade. Pelareorep in combination with pembrolizumab and single-agent chemotherapy has since resulted in disease control in three of five evaluable patients in relapsed metastatic PDAC patients with a manageable safety profile (NCT02620423) (Table 3) [99].

Adoptive T-cell transfer techniques are also being investigated in the context of PDAC. Chimeric antigen receptor $\mathrm{T}$-cell (CAR-T) therapy, which uses T-cells engineered to recognise a specific tumour antigen, appears to be the most effective method available. CAR-T therapy targeted to MUC1, a neoantigen expression in a variety of cancers including PDAC, has been shown to demonstrate target-specific cytotoxicity and improved survival in xenograft models of PDAC [100], with a phase I/II clinical study currently recruiting (NCT02587689). Other targets under clinical evaluation are mesothelin and carcinoembryonic antigen (NCT02465983, NCT02349724), the former having preliminary data suggesting activity in patients with PDAC [101].

\section{Conclusion}

The systemic treatment of advanced PDAC remains challenging. Although clinical trials have led to incremental gains in a survival benefit in recent years, significant improvements in patient outcomes have remained elusive for decades. Developing methods to improve the resolution of patient selection is crucial to exploit the benefit derived from existing therapies while we wait for newer therapies that target stromal elements or the TME to be fully evaluated and approved. Simultaneously, further translational and clinical work will be essential to advance our understanding of novel therapeutic targets and methods of manipulating the immune microenvironment to ensure the successful translation of rational precision therapeutics into the clinic. Finally, combinatorial strategies supported by sound biological rationale hold potential to fulfil the persistent unmet need posed by this aggressive malignancy.

\section{Conflicts of Interest}

Caroline Fong and Emma Burke have no conflicting interests to declare, commercial, intellectual, or otherwise.

\section{Acknowledgments}

David Cunningham receives research funding from AstraZeneca, Celgene, MedImmune, Bayer, 4SC, Clovis, Eli Lilly, Janssen, and Merck. Naureen Starling has received research funding from AstraZeneca, Bristol-Myers Squibb, and Pfizer; travel and accommodation from AstraZeneca, Bristol-Myers Squibb, Eli Lilly, Merck, and Roche; and honoraria from AstraZeneca, Eli Lilly, Merck, and Servier. Naureen Starling is on the advisory board for Pfizer, AstraZeneca, and Servier. This work is supported by the National Institute for Health Research (NIHR) Biomedical Research Centre (BRC) at The Royal Marsden NHS Foundation Trust and the Institute of Cancer Research.

\section{References}

[1] F. Bray, J. Ferlay, I. Soerjomataram, R. L. Siegel, L. A. Torre, and A. Jemal, "Global cancer statistics 2018: GLOBOCAN estimates of incidence and mortality worldwide for 36 cancers in 185 countries," CA: A Cancer Journal for Clinicians, vol. 68, no. 6, pp. 394-424, 2018.

[2] J. P. Neoptolemos, D. H. Palmer, P. Ghaneh et al., "Comparison of adjuvant gemcitabine and capecitabine with gemcitabine monotherapy in patients with resected pancreatic cancer (ESPAC-4): a multicentre, open-label, randomised, phase 3 trial," The Lancet, vol. 389, no. 10073, pp. 1011-1024, 2017.

[3] K. Uesaka, N. Boku, A. Fukutomi et al., "Adjuvant chemotherapy of S-1 versus gemcitabine for resected pancreatic cancer: a phase 3, open-label, randomised, non-inferiority trial (JASPAC 01)," The Lancet, vol. 388, no. 10041, pp. 248-257, 2016.

[4] T. Conroy, P. Hammel, M. Hebbar et al., "FOLFIRINOX or gemcitabine as adjuvant therapy for pancreatic cancer," The New England Journal of Medicine, vol. 379, no. 25, pp. 2395-2406, 2018.

[5] "Pancreatic cancer survival statistics | Cancer Research UK," https://www.cancerresearchuk.org/health-professional/ cancer-statistics/statistics-by-cancer-type/pancreatic-cancer/ survival\#heading-Zero.

[6] T. Conroy, F. Desseigne, M. Ychou et al., "FOLFIRINOX versus gemcitabine for metastatic pancreatic cancer," The New England Journal of Medicine, vol. 364, no. 19, pp. 18171825, 2011.

[7] L. Rahib, B. D. Smith, R. Aizenberg, A. B. Rosenzweig, J. M. Fleshman, and L. M. Matrisian, "Projecting cancer incidence and deaths to 2030: the unexpected burden of thyroid, liver, and pancreas cancers in the United States," Cancer Research, vol. 74, no. 11, pp. 2913-2921, 2014.

[8] H. A. Burris 3rd, M. J. Moore, J. Andersen et al., "Improvements in survival and clinical benefit with gemcitabine as first-line therapy for patients with advanced pancreas cancer: a randomized trial," Journal of Clinical Oncology, vol. 15, no. 6, pp. 2403-2413, 1997.

[9] C. Louvet, R. Labianca, P. Hammel et al., "Gemcitabine in combination with oxaliplatin compared with gemcitabine alone in locally advanced or metastatic pancreatic cancer: results of a GERCOR and GISCAD phase III trial," Journal of Clinical Oncology, vol. 23, no. 15, pp. 3509-3516, 2005.

[10] C. M. Rocha Lima, M. R. Green, R. Rotche et al., "Irinotecan plus gemcitabine results in no survival advantage compared with gemcitabine monotherapy in patients with locally advanced or metastatic pancreatic cancer despite increased tumor response rate," Journal of Clinical Oncology, vol. 22, no. 18, pp. 3776-3783, 2004.

[11] H. Oettle, D. Richards, R. K. Ramanathan et al., "A phase III trial of pemetrexed plus gemcitabine versus gemcitabine in patients with unresectable or metastatic pancreatic cancer," Annals of Oncology, vol. 16, no. 10, pp. 1639-1645, 2005.

[12] H. L. Kindler, T. Ioka, D. J. Richel et al., "Axitinib plus gemcitabine versus placebo plus gemcitabine in patients with advanced pancreatic adenocarcinoma: a double-blind randomised phase 3 study," The Lancet Oncology, vol. 12, no. 3, pp. 256-262, 2011.

[13] M. J. Moore, D. Goldstein, J. Hamm et al., "Erlotinib plus gemcitabine compared with gemcitabine alone in patients 
with advanced pancreatic cancer: a phase III trial of the National Cancer Institute of Canada Clinical Trials Group," Journal of Clinical Oncology, vol. 25, no. 15, pp. 1960-1966, 2007.

[14] D. Cunningham, I. Chau, D. D. Stocken et al., "Phase III randomized comparison of gemcitabine versus gemcitabine plus capecitabine in patients with advanced pancreatic cancer," Journal of Clinical Oncology, vol. 27, no. 33, pp. 5513-5518, 2009.

[15] D. D. Von Hoff, T. Ervin, F. P. Arena et al., "Increased survival in pancreatic cancer with nab-paclitaxel plus gemcitabine," The New England Journal of Medicine, vol. 369, no. 18, pp. 1691-1703, 2013.

[16] S. Pusceddu, M. Ghidini, M. Torchio et al., "Comparative effectiveness of gemcitabine plus nab-paclitaxel and FOLFIRINOX in the first-line setting of metastatic pancreatic cancer: a systematic review and meta-analysis," Cancers, vol. 11, no. 4 , p. 484, 2019.

[17] E. G. Chiorean, D. D. von Hoff, J. Tabernero et al., "Secondline therapy after nab-paclitaxel plus gemcitabine or after gemcitabine for patients with metastatic pancreatic cancer," British Journal of Cancer, vol. 115, no. 2, pp. 188-194, 2016.

[18] H. Oettle, H. Riess, J. M. Stieler et al., "Second-line oxaliplatin, folinic acid, and fluorouracil versus folinic acid and fluorouracil alone for gemcitabine-refractory pancreatic cancer: outcomes from the CONKO-003 trial," Journal of Clinical Oncology, vol. 32, no. 23, pp. 2423-2429, 2014.

[19] S. Gill, Y. J. Ko, C. Cripps et al., "PANCREOX: a randomized phase III study of fluorouracil/leucovorin with or without oxaliplatin for second-line advanced pancreatic cancer in patients who have received gemcitabine-based chemotherapy," Journal of Clinical Oncology, vol. 34, no. 32, pp. 39143920, 2016.

[20] A. Wang-Gillam, C. P. Li, G. Bodoky et al., "Nanoliposomal irinotecan with fluorouracil and folinic acid in metastatic pancreatic cancer after previous gemcitabine-based therapy (NAPOLI-1): a global, randomised, open-label, phase 3 trial," The Lancet, vol. 387, no. 10018, pp. 545-557, 2016.

[21] A. Wang-Gillam, R. A. Hubner, J. T. Siveke et al., "NAPOLI-1 phase 3 study of liposomal irinotecan in metastatic pancreatic cancer: final overall survival analysis and characteristics of long-term survivors," European Journal of Cancer, vol. 108, pp. 78-87, 2019.

[22] C. Almoguera, D. Shibata, K. Forrester, J. Martin, N. Arnheim, and M. Perucho, "Most human carcinomas of the exocrine pancreas contain mutant c-K-ras genes," Cell, vol. 53, no. 4, pp. 549-554, 1988.

[23] D. Zeitouni, Y. Pylayeva-Gupta, C. der, and K. Bryant, "KRAS mutant pancreatic cancer: no lone path to an effective treatment," Cancers, vol. 8, no. 4, p. 45, 2016.

[24] E. Van Cutsem, H. van de Velde, P. Karasek et al., "Phase III trial of gemcitabine plus tipifarnib compared with gemcitabine plus placebo in advanced pancreatic cancer," Journal of Clinical Oncology, vol. 22, no. 8, pp. 1430-1438, 2004.

[25] G. Bodoky, C. Timcheva, D. R. Spigel et al., "A phase II openlabel randomized study to assess the efficacy and safety of selumetinib (AZD6244 [ARRY-142886]) versus capecitabine in patients with advanced or metastatic pancreatic cancer who have failed first-line gemcitabine therapy," Investigational New Drugs, vol. 30, no. 3, pp. 1216-1223, 2012.

[26] J. R. Infante, B. G. Somer, J. O. Park et al., "A randomised, double-blind, placebo-controlled trial of trametinib, an oral
MEK inhibitor, in combination with gemcitabine for patients with untreated metastatic adenocarcinoma of the pancreas," European Journal of Cancer, vol. 50, no. 12, pp. 2072-2081, 2014.

[27] D. B. Zhen, A. Coveler, S. Zanon, M. Reni, and E. G. Chiorean, "Biomarker-driven and molecularly targeted therapies for pancreatic adenocarcinoma," Seminars in Oncology, vol. 45, no. 3, pp. 107-115, 2018.

[28] P. A. Philip, J. Benedetti, C. L. Corless et al., "Phase III study comparing gemcitabine plus cetuximab versus gemcitabine in patients with advanced pancreatic adenocarcinoma: southwest oncology group-directed intergroup trial S0205," Journal of Clinical Oncology, vol. 28, no. 22, pp. 3605-3610, 2010.

[29] R. A. Moffitt, R. Marayati, E. L. Flate et al., "Virtual microdissection identifies distinct tumor- and stroma-specific subtypes of pancreatic ductal adenocarcinoma," Nature Genetics, vol. 47, no. 10, pp. 1168-1178, 2015.

[30] E. S. Knudsen, E. M. O’Reilly, J. R. Brody, and A. K. Witkiewicz, "Genetic diversity of pancreatic ductal adenocarcinoma and opportunities for precision medicine," Gastroenterology, vol. 150, no. 1, pp. 48-63, 2016.

[31] N. Waddell, M. Pajic, A.-M. Patch et al., "Whole genomes redefine the mutational landscape of pancreatic cancer," Nature, vol. 518, no. 7540, pp. 495-501, 2015.

[32] P. Bailey, D. K. Chang, K. Nones et al., "Genomic analyses identify molecular subtypes of pancreatic cancer," Nature, vol. 531, no. 7592, pp. 47-52, 2016.

[33] E. A. Collisson, A. Sadanandam, P. Olson et al., "Subtypes of pancreatic ductal adenocarcinoma and their differing responses to therapy," Nature Medicine, vol. 17, no. 4, pp. 500-503, 2011.

[34] K. L. Aung, S. E. Fischer, R. E. Denroche et al., "Genomicsdriven precision medicine for advanced pancreatic cancer: early results from the COMPASS trial," Clinical Cancer Research, vol. 24, no. 6, pp. 1344-1354, 2018.

[35] A. L. Bredenoord, H. Clevers, and J. A. Knoblich, "Human tissues in a dish: the research and ethical implications of organoid technology," Science, vol. 355, no. 6322, article eaaf9414, 2017.

[36] G. Vlachogiannis, S. Hedayat, A. Vatsiou et al., "Patientderived organoids model treatment response of metastatic gastrointestinal cancers," Science, vol. 359, no. 6378, pp. 920 926, 2018.

[37] H. Tiriac, P. Belleau, D. D. Engle et al., “Organoid profiling identifies common responders to chemotherapy in pancreatic cancer," Cancer Discovery, vol. 8, no. 9, pp. 1112-1129, 2018.

[38] "Home - Precision Panc | Precision Panc," http://www .precisionpanc.org/.

[39] D. Ford, D. F. Easton, D. T. Bishop, S. A. Narod, and D. E. Goldgar, "Risks of cancer in BRCA1-mutation carriers," The Lancet, vol. 343, no. 8899, pp. 692-695, 1994.

[40] J. B. Greer and D. C. Whitcomb, "Role of BRCA1 and BRCA2 mutations in pancreatic cancer," Gut, vol. 56, no. 5, pp. 601605, 2007.

[41] S. Holter, A. Borgida, A. Dodd et al., "Germline BRCA mutations in a large clinic-based cohort of patients with pancreatic adenocarcinoma," Journal of Clinical Oncology, vol. 33, no. 28, pp. 3124-3129, 2015.

[42] M. C. Villarroel, N. V. Rajeshkumar, I. Garrido-Laguna et al., "Personalizing cancer treatment in the age of global genomic 
analyses: PALB2 gene mutations and the response to DNA damaging agents in pancreatic cancer," Molecular Cancer Therapeutics, vol. 10, no. 1, pp. 3-8, 2011.

[43] L. Perkhofer, A. Schmitt, M. C. Romero Carrasco et al., "ATM deficiency generating genomic instability sensitizes pancreatic ductal adenocarcinoma cells to therapy-induced DNA damage," Cancer Research, vol. 77, no. 20, pp. 55765590, 2017.

[44] J. Smith, L. Mun Tho, N. Xu, and D. A. Gillespie, "Chapter 3 The ATM-Chk2 and ATR-Chk1 pathways in DNA damage signaling and cancer," Advances in Cancer Research, vol. 108, pp. 73-112, 2010.

[45] D. Li, M. Frazier, D. B. Evans et al., "Single nucleotide polymorphisms of RecQ1, RAD54L, and ATM genes are associated with reduced survival of pancreatic cancer," Journal of Clinical Oncology, vol. 24, no. 11, pp. 1720-1728, 2006.

[46] T. Golan, P. Hammel, M. Reni et al., "Maintenance olaparib for germline BRCA-mutated metastatic pancreatic cancer," New England Journal of Medicine, vol. 381, no. 4, pp. 317327, 2019.

[47] C. J. Whatcott, C. H. Diep, P. Jiang et al., "Desmoplasia in primary tumors and metastatic lesions of pancreatic cancer," Clinical Cancer Research, vol. 21, no. 15, pp. 3561-3568, 2015.

[48] M. F. B. Nielsen, M. B. Mortensen, and S. Detlefsen, "Key players in pancreatic cancer-stroma interaction: cancerassociated fibroblasts, endothelial and inflammatory cells," World Journal of Gastroenterology, vol. 22, no. 9, p. 2678, 2016.

[49] J. Kleeff, P. Beckhove, I. Esposito et al., "Pancreatic cancer microenvironment," International Journal of Cancer, vol. 121, no. 4, pp. 699-705, 2007.

[50] B. C. Özdemir, T. Pentcheva-Hoang, J. L. Carstens et al., "Depletion of carcinoma-associated fibroblasts and fibrosis induces immunosuppression and accelerates pancreas cancer with reduced survival," Cancer Cell, vol. 25, no. 6, pp. 719734, 2014.

[51] A. D. Rhim, P. E. Oberstein, D. H. Thomas et al., "Stromal elements act to restrain, rather than support, pancreatic ductal adenocarcinoma," Cancer Cell, vol. 25, no. 6, pp. 735-747, 2014.

[52] C. Neuzillet, A. Tijeras-Raballand, C. Ragulan et al., "Interand intra-tumoural heterogeneity in cancer-associated fibroblasts of human pancreatic ductal adenocarcinoma," The Journal of Pathology, vol. 248, no. 1, pp. 51-65, 2019.

[53] L. B. Alexandrov, S. Nik-Zainal, D. C. Wedge et al., "Signatures of mutational processes in human cancer," Nature, vol. 500, no. 7463, pp. 415-421, 2013.

[54] P. Bailey, D. K. Chang, M. A. Forget et al., "Exploiting the neoantigen landscape for immunotherapy of pancreatic ductal adenocarcinoma," Scientific Reports, vol. 6, no. 1, article 35848, 2016.

[55] V. P. Balachandran, M. Łuksza, J. N. Zhao et al., "Identification of unique neoantigen qualities in long-term survivors of pancreatic cancer," Nature, vol. 551, no. 7681, pp. 512516, 2017.

[56] A. Fukunaga, M. Miyamoto, Y. Cho et al., "CD8+ tumorinfiltrating lymphocytes together with CD4+ tumorinfiltrating lymphocytes and dendritic cells improve the prognosis of patients with pancreatic adenocarcinoma," Pancreas, vol. 28, no. 1, pp. e26-e31, 2004.
[57] K. P. Olive, M. A. Jacobetz, C. J. Davidson et al., "Inhibition of hedgehog signaling enhances delivery of chemotherapy in a mouse model of pancreatic cancer," Science, vol. 324, no. 5933, pp. 1457-1461, 2009.

[58] J. J. Lee, R. M. Perera, H. Wang et al., "Stromal response to hedgehog signaling restrains pancreatic cancer progression," Proceedings of the National Academy of Sciences of the United States of America, vol. 111, no. 30, pp. E3091-E3100, 2014.

[59] H. Jiang, S. Hegde, B. L. Knolhoff et al., "Targeting focal adhesion kinase renders pancreatic cancers responsive to checkpoint immunotherapy," Nature Medicine, vol. 22, no. 8, pp. 851-860, 2016.

[60] A. Begum, T. Ewachiw, C. Jung et al., "The extracellular matrix and focal adhesion kinase signaling regulate cancer stem cell function in pancreatic ductal adenocarcinoma," PLoS One, vol. 12, no. 7, article e0180181, 2017.

[61] I. M. Shapiro, V. N. Kolev, C. M. Vidal et al., "Merlin deficiency predicts FAK inhibitor sensitivity: a synthetic lethal relationship," Science Translational Medicine, vol. 6, no. 237, article 237ra68, 2014.

[62] J. C. Soria, H. K. Gan, S. P. Blagden et al., "A phase I, pharmacokinetic and pharmacodynamic study of GSK2256098, a focal adhesion kinase inhibitor, in patients with advanced solid tumors," Annals of Oncology, vol. 27, no. 12, pp. 2268-2274, 2016.

[63] M. Quan, J. Cui, T. Xia et al., "Merlin/NF2 suppresses pancreatic tumor growth and metastasis by attenuating the FOXM1-mediated Wnt/ $\beta$-catenin signaling," Cancer Research, vol. 75, no. 22, pp. 4778-4789, 2015.

[64] B. P. Toole and M. G. Slomiany, "Hyaluronan: a constitutive regulator of chemoresistance and malignancy in cancer cells," Seminars in Cancer Biology, vol. 18, no. 4, pp. 244-250, 2008.

[65] P. P. Provenzano, C. Cuevas, A. E. Chang, V. K. Goel, D. D. von Hoff, and S. R. Hingorani, "Enzymatic targeting of the stroma ablates physical barriers to treatment of pancreatic ductal adenocarcinoma," Cancer Cell, vol. 21, no. 3, pp. 418-429, 2012.

[66] M. A. Jacobetz, D. S. Chan, A. Neesse et al., "Hyaluronan impairs vascular function and drug delivery in a mouse model of pancreatic cancer," Gut, vol. 62, no. 1, pp. 112 120, 2016.

[67] S. R. Hingorani, L. Zheng, A. J. Bullock et al., "HALO 202: randomized phase II study of PEGPH20 plus nab-paclitaxel/gemcitabine versus nab-paclitaxel/gemcitabine in patients with untreated, metastatic pancreatic ductal adenocarcinoma," Journal of Clinical Oncology, vol. 36, no. 4, pp. 359366, 2018.

[68] R. K. Ramanathan, S. L. McDonough, P. A. Philip et al., "Phase IB/II randomized study of FOLFIRINOX plus pegylated recombinant human hyaluronidase versus FOLFIRINOX alone in patients with metastatic pancreatic adenocarcinoma: SWOG S1313," Journal of Clinical Oncology, vol. 37, no. 13, pp. 1062-1069, 2019.

[69] R. E. Royal, C. Levy, K. Turner et al., "Phase 2 trial of single agent ipilimumab (anti-CTLA-4) for locally advanced or metastatic pancreatic adenocarcinoma," Journal of Immunotherapy, vol. 33, no. 8, pp. 828-833, 2010.

[70] J. R. Brahmer, S. S. Tykodi, L. Q. M. Chow et al., "Safety and activity of anti-PD-L1 antibody in patients with advanced cancer," The New England Journal of Medicine, vol. 366, no. 26, pp. 2455-2465, 2012. 
[71] E. M. O'Reilly, D.-Y. Oh, N. Dhani et al., "A randomized phase 2 study of durvalumab monotherapy and in combination with tremelimumab in patients with metastatic pancreatic ductal adenocarcinoma (mPDAC): ALPS study," Journal of Clinical Oncology, vol. 36, 4_Supplement, p. 217, 2018.

[72] D. T. Le, J. N. Uram, H. Wang et al., "PD-1 blockade in tumors with mismatch repair deficiency," The New England Journal of Medicine, vol. 372, no. 26, pp. 2509-2520, 2015.

[73] D. T. Le, J. N. Durham, K. N. Smith et al., "Mismatch repair deficiency predicts response of solid tumors to PD-1 blockade," Science, vol. 357, no. 6349, pp. 409-413, 2017.

[74] J. L. Humphris, A. M. Patch, K. Nones et al., "Hypermutation in pancreatic cancer," Gastroenterology, vol. 152, no. 1, pp. 68-74.e2, 2017.

[75] A. M. McDonnell, W. J. Lesterhuis, A. Khong et al., “Tumorinfiltrating dendritic cells exhibit defective cross-presentation of tumor antigens, but is reversed by chemotherapy," European Journal of Immunology, vol. 45, no. 1, pp. 49-59, 2015.

[76] N. M. Haynes, R. G. van der Most, R. A. Lake, and M. J. Smyth, "Immunogenic anti-cancer chemotherapy as an emerging concept," Current Opinion in Immunology, vol. 20, no. 5, pp. 545-557, 2008.

[77] P. C. Hermann, S. L. Huber, T. Herrler et al., "Distinct populations of cancer stem cells determine tumor growth and metastatic activity in human pancreatic cancer," Cell Stem Cell, vol. 1, no. 3, pp. 313-323, 2007.

[78] C. Feig, J. O. Jones, M. Kraman et al., "Targeting CXCL12 from FAP-expressing carcinoma-associated fibroblasts synergizes with anti-PD-L1 immunotherapy in pancreatic cancer," Proceedings of the National Academy of Sciences of the United States of America, vol. 110, no. 50, pp. 2021220217, 2013.

[79] M. Hidalgo, R. Epelbaum, B. M. Wolpin et al., “1133PD A phase IIa trial to assess the safety and efficacy of BL-8040 and pembrolizumab in patients with metastatic pancreatic adenocarcinoma (PDAC)," Annals of Oncology, vol. 29, article mdy288.006, 8 Supplement, 2018.

[80] J. B. Mitchem, D. J. Brennan, B. L. Knolhoff et al., "Targeting tumor-infiltrating macrophages decreases tumor-initiating cells, relieves immunosuppression, and improves chemotherapeutic responses," Cancer Research, vol. 73, no. 3, pp. 11281141, 2013.

[81] Y. Zhu, B. L. Knolhoff, M. A. Meyer et al., "CSF1/CSF1R blockade reprograms tumor-infiltrating macrophages and improves response to T-cell checkpoint immunotherapy in pancreatic cancer models," Cancer Research, vol. 74, no. 18, pp. 5057-5069, 2014.

[82] J. P. Morton and O. J. Sansom, "CXCR2 inhibition in pancreatic cancer: opportunities for immunotherapy?," Immunotherapy, vol. 9, no. 1, pp. 9-12, 2017.

[83] D. J. Nicholls, K. Wiley, I. Dainty et al., "Pharmacological characterization of AZD5069, a slowly reversible CXC chemokine receptor 2 antagonist," The Journal of Pharmacology and Experimental Therapeutics, vol. 353, no. 2, pp. 340-350, 2015.

[84] C. W. Steele, S. A. Karim, J. D. G. Leach et al., "CXCR2 inhibition profoundly suppresses metastases and augments immunotherapy in pancreatic ductal adenocarcinoma," Cancer Cell, vol. 29, no. 6, pp. 832-845, 2016.

[85] D. Masso-Valles, T. Jauset, E. Serrano et al., "Ibrutinib exerts potent antifibrotic and antitumor activities in mouse models of pancreatic adenocarcinoma," Cancer Research, vol. 75, no. 8, pp. 1675-1681, 2015.

[86] A. J. Gunderson, M. M. Kaneda, T. Tsujikawa et al., "Bruton tyrosine kinase-dependent immune cell cross-talk drives pancreas cancer," Cancer Discovery, vol. 6, no. 3, pp. 270285, 2016.

[87] M. Tempero, D. Oh, T. Macarulla et al., "Ibrutinib in combination with nab-paclitaxel and gemcitabine as first-line treatment for patients with metastatic pancreatic adenocarcinoma: results from the phase 3 RESOLVE study," Annals of Oncology, vol. 30, article mdz154.001, 4 Supplement, 2019.

[88] H. Yanagimoto, H. Shiomi, S. Satoi et al., "A phase II study of personalized peptide vaccination combined with gemcitabine for non-resectable pancreatic cancer patients," Oncology Reports, vol. 24, no. 3, pp. 795-801, 2010.

[89] L. Xia, D. S. Schrump, and J. C. Gildersleeve, "Whole-cell cancer vaccines induce large antibody responses to carbohydrates and glycoproteins," Cell Chemical Biology, vol. 23, no. 12, pp. 1515-1525, 2016.

[90] E. R. Lutz, A. A. Wu, E. Bigelow et al., "Immunotherapy converts nonimmunogenic pancreatic tumors into immunogenic foci of immune regulation," Cancer Immunology Research, vol. 2, no. 7, pp. 616-631, 2014.

[91] K. C. Soares, A. A. Rucki, A. A. Wu et al., "PD-1/PD-L1 blockade together with vaccine therapy facilitates effector Tcell infiltration into pancreatic tumors," Journal of Immunotherapy, vol. 38, no. 1, pp. 1-11, 2015.

[92] D. T. Le, A. Wang-Gillam, V. Picozzi et al., "Safety and survival with GVAX pancreas prime and Listeria Monocytogenes-expressing mesothelin (CRS-207) boost vaccines for metastatic pancreatic cancer," Journal of Clinical Oncology, vol. 33, no. 12, pp. 1325-1333, 2015.

[93] D. T. Le, A. H. Ko, Z. A. Wainberg et al., "Results from a phase $2 \mathrm{~b}$, randomized, multicenter study of GVAX pancreas and CRS-207 compared to chemotherapy in adults with previously-treated metastatic pancreatic adenocarcinoma (ECLIPSE Study)," Journal of Clinical Oncology, vol. 35, 4 Supplement, p. 345, 2017.

[94] S. Pushalkar, M. Hundeyin, D. Daley et al., "The pancreatic cancer microbiome promotes oncogenesis by induction of innate and adaptive immune suppression," Cancer Discovery, vol. 8, no. 4, pp. 403-416, 2018.

[95] E. A. Chiocca and S. D. Rabkin, "Oncolytic viruses and their application to cancer immunotherapy," Cancer Immunology Research, vol. 2, no. 4, pp. 295-300, 2014.

[96] S. Mulvihill, R. Warren, A. Venook et al., "Safety and feasibility of injection with an E1B-55 kDa gene-deleted, replicationselective adenovirus (ONYX-015) into primary carcinomas of the pancreas: a phase I trial," Gene Therapy, vol. 8, no. 4, pp. 308-315, 2001.

[97] J. R. Hecht, R. Bedford, J. L. Abbruzzese et al., "A phase I/II trial of intratumoral endoscopic ultrasound injection of ONYX-015 with intravenous gemcitabine in unresectable pancreatic carcinoma," Clinical Cancer Research, vol. 9, no. 2, pp. 555-561, 2003.

[98] D. Mahalingam, S. Goel, S. Aparo et al., "A phase ii study of pelareorep (REOLYSIN ${ }^{\circledR}$ ) in combination with gemcitabine for patients with advanced pancreatic adenocarcinoma," Cancers, vol. 10, no. 6, p. 160, 2018.

[99] D. Mahalingam, C. Fountzilas, J. L. Moseley et al., “A study of pelareorep in combination with pembrolizumab and 
chemotherapy in patients (pts) with relapsed metastatic adenocarcinoma of the pancreas (MAP)," Journal of Clinical Oncology, vol. 36, 4_Supplement, p. 283, 2018.

[100] A. D. Posey Jr., R. D. Schwab, A. C. Boesteanu et al., "Engineered CAR $\mathrm{T}$ cells targeting the cancer-associated Tnglycoform of the membrane mucin MUC1 control adenocarcinoma," Immunity, vol. 44, no. 6, pp. 1444-1454, 2016.

[101] G. L. Beatty, M. H. O’Hara, S. F. Lacey et al., “Activity of mesothelin-specific chimeric antigen receptor T cells against pancreatic carcinoma metastases in a phase 1 trial," Gastroenterology, vol. 155, no. 1, pp. 29-32, 2018.

[102] D. V. T. Catenacci, M. R. Junttila, T. Karrison et al., "Randomized phase Ib/II study of gemcitabine plus placebo or vismodegib, a hedgehog pathway inhibitor, in patients with metastatic pancreatic cancer," Journal of Clinical Oncology, vol. 33, no. 36, pp. 4284-4292, 2015.

[103] A. H. Ko, N. LoConte, M. A. Tempero et al., "A phase I study of FOLFIRINOX plus IPI-926, a hedgehog pathway inhibitor, for Advanced Pancreatic Adenocarcinoma," Pancreas, vol. 45, no. 3, pp. 370-375, 2016.

[104] D. A. Richards, J. Stephenson, B. M. Wolpin et al., "A phase Ib trial of IPI-926, a hedgehog pathway inhibitor, plus gemcitabine in patients with metastatic pancreatic cancer," Journal of Clinical Oncology, vol. 30, 4_Supplement, p. 213, 2012.

[105] K. L. Aung, E. McWhirter, S. Welch et al., "A phase II trial of GSK2256098 and trametinib in patients with advanced pancreatic ductal adenocarcinoma (PDAC) (MOBILITY-002 Trial, NCT02428270)," Journal of Clinical Oncology, vol. 36, 4_Supplement, p. 409, 2018.

[106] A. Wang-Gillam, A. C. Lockhart, B. R. Tan et al., "Phase I study of defactinib combined with pembrolizumab and gemcitabine in advanced cancer," Journal of Clinical Oncology, vol. 35, article TPS505, 4_Supplement, 2017.

[107] A. H. Ko, E. G. Chiorean, E. L. Kwak et al., "Final results of a phase Ib dose-escalation study of PRI-724, a CBP/betacatenin modulator, plus gemcitabine (GEM) in patients with advanced pancreatic adenocarcinoma (APC) as second-line therapy after FOLFIRINOX or FOLFOX," Journal of Clinical Oncology, vol. 34, article e15721, 15_Supplement, 2016.

[108] E. Dotan, D. B. Cardin, H. J. Lenz et al., "Phase Ib study of WNT inhibitor ipafricept (IPA) with nab-paclitaxel (Nab-P) and gemcitabine $(\mathrm{G})$ in patients (pts) with previously untreated stage IV pancreatic cancer (mPC)," Journal of Clinical Oncology, vol. 37, 4_Supplement, p. 369, 2019.

[109] E. Van Cutsem, A. E. Hendifar, M. Reni et al., "Global phase 3 , randomized, double-blind, placebo-controlled study evaluating PEGylated recombinant human hyaluronidase $\mathrm{PH} 20$ (PEGPH20) plus nab-paclitaxel and gemcitabine in patients with previously untreated, hyaluronan (HA)-high, stage IV pancreatic ductal adenocarcinoma," Annals of Oncology, vol. 27, article 715TiP, Supplement_6, 2016.

[110] M. J. Borad, S. G. Reddy, N. Bahary et al., "Randomized phase II trial of gemcitabine plus $\mathrm{TH}-302$ versus gemcitabine in patients with advanced pancreatic cancer," Journal of Clinical Oncology, vol. 33, no. 13, pp. 1475-1481, 2015.

[111] E. Van Cutsem, H.-J. Lenz, J. Furuse et al., "MAESTRO: a randomized, double-blind phase III study of evofosfamide (Evo) in combination with gemcitabine (Gem) in previously untreated patients (pts) with metastatic or locally advanced unresectable pancreatic ductal adenocarcinoma (PDAC),"
Journal of Clinical Oncology, vol. 34, article 4007, 15_Supplement, 2016.

[112] H. L. Kindler, D. Niedzwiecki, D. Hollis et al., "Gemcitabine plus bevacizumab compared with gemcitabine plus placebo in patients with advanced pancreatic cancer: phase III trial of the cancer and leukemia group B (CALGB 80303)," Journal of Clinical Oncology, vol. 28, no. 22, pp. 3617-3622, 2010.

[113] K. Perez, D. A. Rubinson, J. M. Cleary et al., "Phase 1b study of gemcitabine, nab-paclitaxel, and ficlatuzumab in patients with advanced pancreatic cancer," Journal of Clinical Oncology, vol. 36, article TPS4152, 15_Supplement, 2018.

[114] D. J. Renouf, N. C. Dhani, P. Kavan et al., "The Canadian Cancer Trials Group PA.7 trial: results from the safety run in of a randomized phase II study of gemcitabine (GEM) and nab-paclitaxel (Nab-P) versus GEM, nab-P, durvalumab (D), and tremelimumab ( $\mathrm{T}$ ) as first-line therapy in metastatic pancreatic ductal adenocarcinoma (mPDAC)," Journal of Clinical Oncology, vol. 36, 4_Supplement, p. 349, 2018.

[115] Z. A. Wainberg, H. S. Hochster, E. J. H. Kim et al., "Phase I study of nivolumab (Nivo) + nab-paclitaxel $(n a b-\mathrm{P})+$ gemcitabine (Gem) in advanced pancreatic cancer (APC)," Journal of Clinical Oncology, vol. 37, 4_Supplement, p. 298, 2019.

[116] A. Wang-Gillam, A. C. Lockhart, B. R. Tan et al., "Phase I study of defactinib combined with pembrolizumab and gemcitabine in patients with advanced cancer," Journal of Clinical Oncology, vol. 36, 4_Supplement, p. 380, 2018.

[117] J. Desai, J. S. Kortmansky, N. H. Segal et al., "MORPHEUS: a phase Ib/II study platform evaluating the safety and clinical efficacy of cancer immunotherapy (CIT)-based combinations in gastrointestinal (GI) cancers," Journal of Clinical Oncology, vol. 37, article TPS467, 4_Supplement, 2019.

[118] M. J. Overman, C. D. Lopez, A. B. Benson et al., "A randomized phase 2 study of the Bruton tyrosine kinase (Btk) inhibitor acalabrutinib alone or with pembrolizumab for metastatic pancreatic cancer (mPC)," Journal of Clinical Oncology, vol. 34, article 4130, 15_Supplement, 2016.

[119] Z. Wainberg, S. Piha-Paul, J. Luke, E. Kim, J. Thompson, and N. Pfanzelter, "First in-human phase 1 dose escalation and expansion of a novel combination, anti-CSF-1 receptor (cabiralizumab) plus anti-PD-1 (nivolumab), in patients with advanced solid tumors," Journal for Immunotherapy of Cancer, vol. 5, no. S3, p. 89, 2017.

[120] D. T. Le, T. S. Crocenzi, J. N. Uram et al., "Randomized phase II study of the safety, efficacy, and immune response of GVAX pancreas vaccine (with cyclophosphamide) and CRS-207 with or without nivolumab in patients with previously treated metastatic pancreatic adenocarcinoma (STELLAR)," Journal of Clinical Oncology, vol. 33, article TPS4148, 15_Supplement, 2015. 


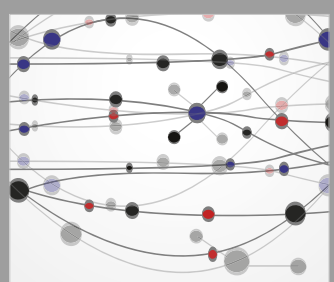

The Scientific World Journal
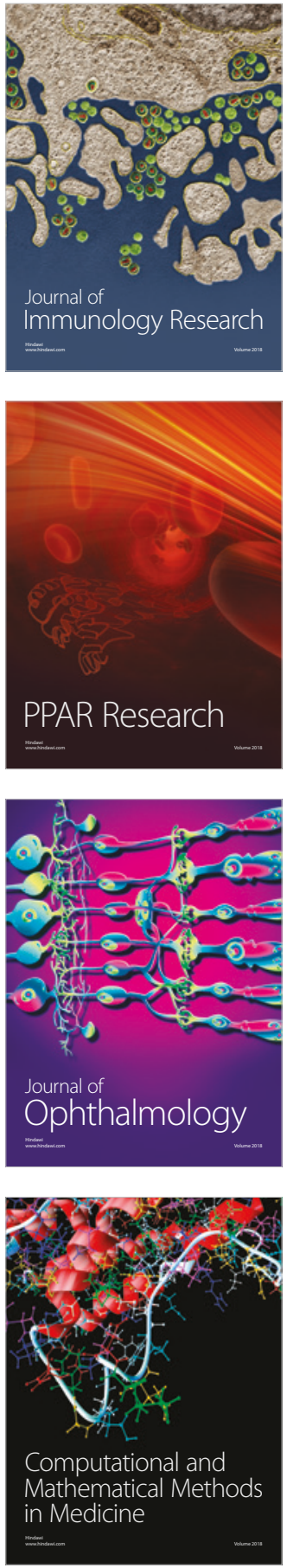

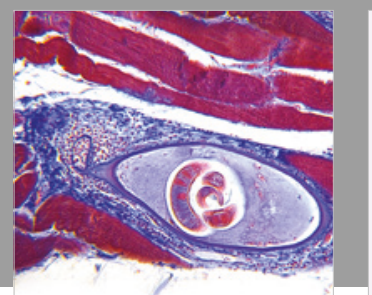

Gastroenterology Research and Practice

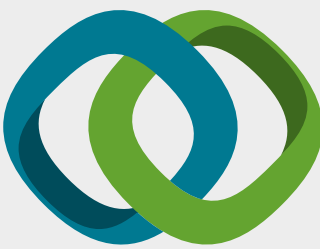

\section{Hindawi}

Submit your manuscripts at

www.hindawi.com
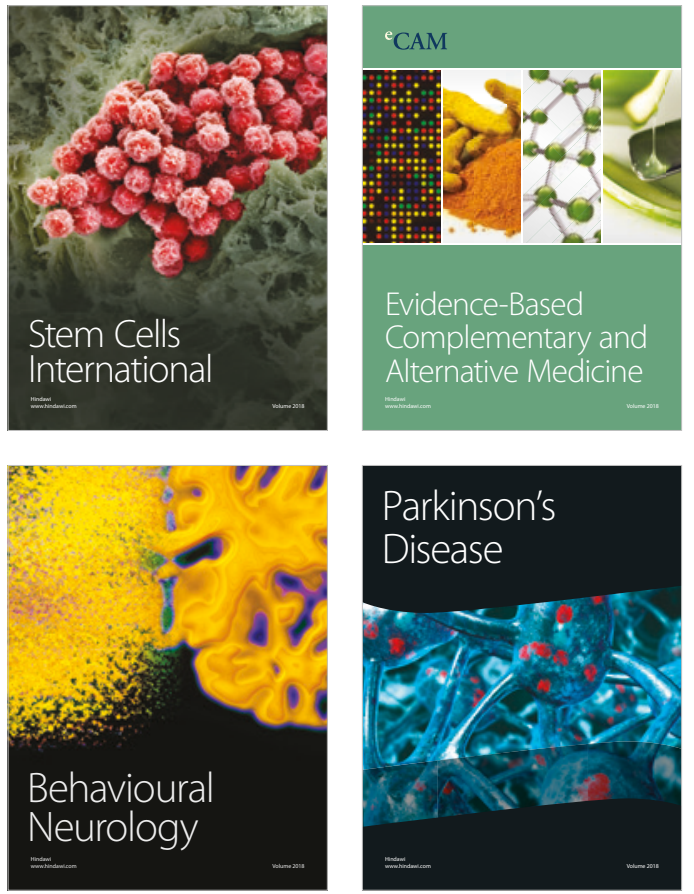

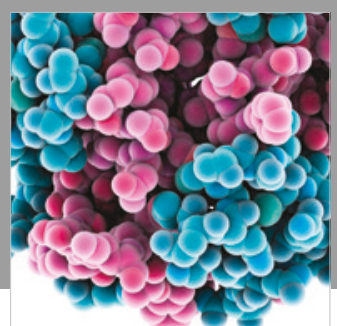

ournal of

Diabetes Research

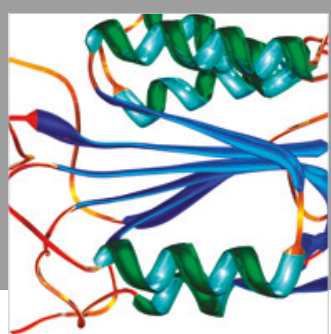

Disease Markers
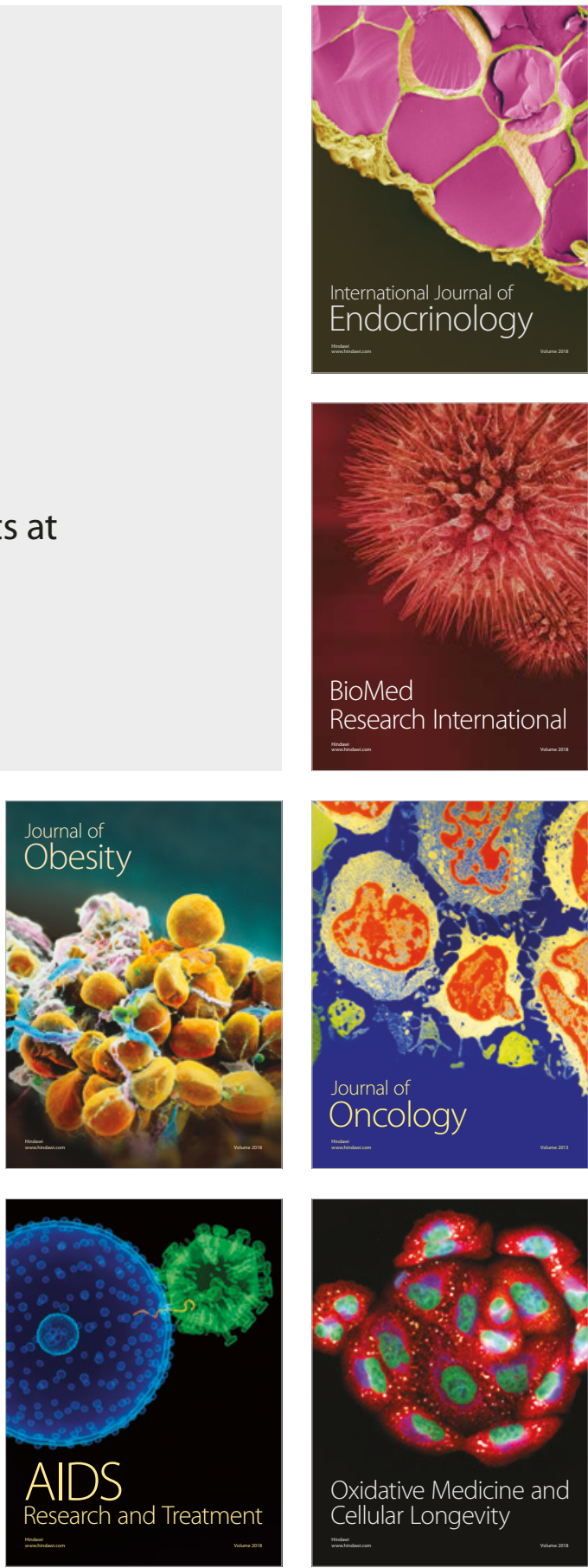